\title{
Asymptotic and Dynamical Analyses of Heat Transfer through a Rectangular Longitudinal Fin
}

\author{
C. Harley \\ Centre for Differential Equations, Continuum Mechanics and Applications, School of Computational and Applied Mathematics, \\ University of the Witwatersrand, Johannesburg, Private Bag 3, Wits 2050, South Africa
}

Correspondence should be addressed to C. Harley; charis.harley@wits.ac.za

Received 12 November 2012; Accepted 19 November 2012

Academic Editor: Fazal M. Mahomed

Copyright (C) 2013 C. Harley. This is an open access article distributed under the Creative Commons Attribution License, which permits unrestricted use, distribution, and reproduction in any medium, provided the original work is properly cited.

The steady heat transfer through a rectangular longitudinal fin is studied. The thermal conductivity and heat transfer coefficient are assumed to be temperature dependent making the resulting ordinary differential equation (ODE) highly nonlinear. An asymptotic solution is used as a means of understanding the relationship between key parameters. A dynamical analysis is also employed for the same purpose.

\section{Introduction}

In this paper we consider the temperature profile in a longitudinal rectangular fin attached to a stationary base surface. Fins are defined as extended surfaces used to enhance the heat dissipation from a hot surface $[1,2]$. They are used in multiple different contexts such as in air-conditioning, air-cooled craft engines, refrigeration, and the cooling of computer processors and oil carrying pipe lines. There are many documented mathematical models which describe the heat transfer in fins of different geometries and profiles with a variety of boundary conditions [1]. Many methods have been implemented as a means of obtaining solutions to such equations, analytical and numerical alike. In much research both the thermal conductivity and heat transfer coefficient have been considered as constants (see e.g., [3-5]). For engineering applications and physical phenomena thermal conductivity of a fin is assumed to be linearly dependent on temperature (see e.g., [6]). In this paper we have chosen the thermal conductivity as a linear function of the temperature and the heat transfer coefficient as a nonlinear function of the temperature.

As far as the author knows, there is no or very little work which has been done on obtaining asymptotic solutions to a problem of the form presented here. An investigation of such solutions is of value given the prevalence of many parameters whose impact and relationship with each other has yet to be fully understood. It is the purpose of asymptotic solutions to reveal the dominant physical mechanisms of the model. In Moitsheki and Harley [7] and Harley and Moitsheki [8], the impact of the thermogeometric parameter was discussed with regards to its proportionality to the length of the fin, $L$. It was noticed that for small values of $\mathscr{M}$ that there seemed to be unstable heat transfer in the fin-this was thought to be related to the fact that $\mathscr{M} \propto$ $L$. Investigating an asymptotic solution to the steady heat transfer in a rectangular longitudinal fin allows us to validate this relationship and more firmly establish the importance of the length of the fin. A dynamical system analysis was conducted in Harley and Moitsheki [8], however, not to the extent conducted here. In this work, the analysis in [8] is improved upon and the behaviour, particularly a the tip of the fin, more meticulously investigated and documented. This dynamical systems analysis also functions as a means of investigating the effect of the thermo-geometric parameter.

\section{Model}

We consider a rectangular longitudinal one-dimensional fin with a cross-sectional area $A_{c}$. The perimeter of the fin is denoted by $P$ and the length of fin by $L$. The fin is attached to a fixed base surface of temperature $T_{b}$ and extends into a fluid of temperature $T_{a}$. The energy balance for a longitudinal 
fin is given by an ordinary differential equation (ODE) [1] as follows:

$$
A_{c} \frac{d}{d X}\left(K(T) \frac{d T}{d X}\right)=P H(T)\left(T-T_{a}\right), \quad 0 \leq X \leq L,
$$

where $K$ and $H$ are the nonuniform thermal conductivity and heat transfer coefficient both of which depend on the temperature (see, e.g., $[3,4,9,10])$. The fin length is measured from the tip to the base as shown in Figure 1 (see also, $[1,3,4]$ ). An insulated fin at one end with the base temperature at the other leads to the following boundary conditions [1]:

$$
T(L)=T_{b},\left.\quad \frac{d T}{d X}\right|_{X=0}=0 .
$$
law [3]

The heat transfer coefficient may be given as the power

$$
H(T)=h_{b}\left(\frac{T-T_{a}}{T_{b}-T_{a}}\right)^{n}
$$

as is done for most industrial applications. Furthermore, the thermal conductivity of the fin may be assumed to vary linearly with temperature for many engineering applications $[3,6]$ and as such may be written as the following:

$$
K(T)=k_{a}\left(1+\lambda\left(T-T_{a}\right)\right)
$$

In the above equations, $H$ is the heat transfer, $h_{b}$ indicates the heat transfer at the base of fin, $K$ is the thermal conductivity, $k_{a}$ is the thermal conductivity of the fin at the ambient temperature, and the exponent $n$ is defined to be dependent upon the heat transfer mode. Here the exponent represents laminar film boiling or condensation when $n=$ $-1 / 4$, laminar natural convection when $n=1 / 4$, turbulent natural convection $n=1 / 3$, nucleate boiling when $n=2$, radiation when $n=3$, and $n$ vanishes for constant heat transfer coefficient. The constant $n$ may vary between -6.6 and 5 , however, in most practical applications it lies between 3 and -3 [11].

Introducing the following dimensionless variables:

$$
\begin{gathered}
x=\frac{X}{L}, \quad \theta=\frac{T-T_{a}}{T_{b}-T_{a}}, \quad h=\frac{H}{h_{b}}, \\
k=\frac{K}{k_{a}}, \quad \mathscr{M}^{2}=\frac{P h_{b} L^{2}}{k_{a} A_{c}},
\end{gathered}
$$

allows (1) to be reduced to the following nondimensional partial differential equation:

$$
\frac{d}{d x}\left[k(\theta) \frac{d \theta}{d x}\right]=\mathscr{M}^{2} \theta h(\theta), \quad 0 \leq x \leq 1 .
$$

In (6) $\theta$ is defined as the dimensionless temperature and $x$ as the dimensionless spatial variable, where $k(\theta)$ is the dimensionless thermal conductivity, $h(\theta)$ is the heat transfer coefficient, and $\mathscr{M}$ is termed the thermo-geometric parameter. In dimensionless variables the heat transfer coefficient is chosen as $h(\theta)=\theta^{n}$ with $n$ termed as the exponent. The thermal conductivity coefficient can be written in dimensionless variables as $k(\theta)=1+\beta \theta$ with $\beta=\lambda\left(T_{b}-\right.$ $T_{a}$ ), where $T_{b}$ is the dimensional fin base temperature and $T_{a}$ is the dimensional ambient temperature. From these choices of $k(\theta)$ and $h(\theta)$, we obtain the one-dimensional nonlinear heat conduction equation:

$$
\frac{d}{d x}\left[(1+\beta \theta) \frac{d \theta}{d x}\right]=\mathscr{M}^{2} \theta^{n+1}, \quad 0 \leq x \leq 1 .
$$

The dimensionless boundary conditions become

$$
\begin{aligned}
& \left.\frac{d \theta}{d x}\right|_{x=0}=0 \quad \text { at the fin tip, } \\
& \theta(1)=1, \quad \text { at the base. }
\end{aligned}
$$

These conditions ensure an insulated fin tip and a constant base temperature.

\section{Asymptotic Analysis for Steady Heat Transfer}

3.1. $\mathscr{M} \ll \epsilon$. In this section we will assume that $\mathscr{M} \ll \epsilon$ (i.e., the thermogeometric parameter is very much smaller than some small parameter $\epsilon$ ) as a means of investigating the nature of the solutions of the steady state case of (7) as follows:

$$
\frac{d}{d x}\left[(1+\beta \theta) \frac{d \theta}{d x}\right]=\mathscr{M}^{2} \theta^{n+1}, \quad 0 \leq x \leq 1, \tau \geq 0,
$$

where $f(x)=1$ with boundary conditions

$$
\begin{gathered}
\left.\frac{\partial \theta}{\partial x}\right|_{x=0}=0 \quad \text { at the fin tip, } \\
\theta(1)=1, \quad \text { at the base. }
\end{gathered}
$$

We assume a solution of the form $\theta(x)=\theta_{0}(x)+\mathscr{M}^{2} \theta_{1}(x)+$ $\mathscr{M}^{4} \theta_{2}(x)$, where $\mathscr{M} \rightarrow 0$. After substitution the term - $\mathscr{M}^{2} \theta(x)^{n+1}$ is expanded using a series expansion so that

$$
\begin{aligned}
- & \mathscr{M}^{2} \theta(x)^{n+1} \\
= & -\mathscr{M}^{2}-(n+1) \theta_{1}(x) \mathscr{M}^{4} \\
& -\left(\frac{1}{2}\left(n^{2}+n\right) \theta_{1}(x)^{2}+(n+1) \theta_{2}(x)\right) \mathscr{M}^{6}+O\left(\mathscr{M}^{7}\right) .
\end{aligned}
$$

In this manner, we are able to separate (11) according to powers of $\mathscr{M}$ and obtain the following system of equations:

$$
\begin{gathered}
O\left(\mathscr{M}^{0}\right): \beta\left(\frac{d \theta_{0}}{d x}\right)^{2}+\frac{d^{2} \theta_{0}}{d x^{2}}\left(1+\beta \theta_{0}\right) \\
O\left(\mathscr{M}^{2}\right):-1+2 \beta \frac{d \theta_{0}}{d x} \frac{d \theta_{1}}{d x}+\beta \theta_{1} \frac{d^{2} \theta_{0}}{d x^{2}}+\frac{d^{2} \theta_{1}}{d x^{2}}\left(1+\beta \theta_{0}\right) \\
O\left(\mathscr{M}^{4}\right):-(n+1) \theta_{1}+\beta\left(\frac{d \theta_{1}}{d x}\right)^{2}+2 \beta \frac{d \theta_{0}}{d x} \frac{d \theta_{2}}{d x} \\
+\beta \theta_{2} \frac{d^{2} \theta_{0}}{d x^{2}}+\beta \theta_{1} \frac{d^{2} \theta_{1}}{d x^{2}}+\frac{d^{2} \theta_{2}}{d x^{2}}\left(1+\beta \theta_{0}\right)
\end{gathered}
$$




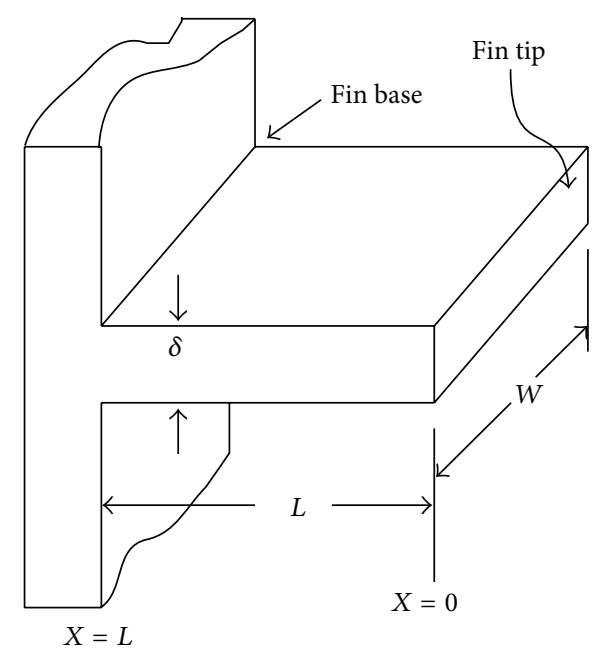

FIGURE 1: Schematic representation of a longitudinal fin with a rectangular profile.

Given these expansions the boundary conditions (10) become

$$
\begin{aligned}
& \left.\frac{\partial \theta_{0}}{\partial x}\right|_{x=0}=\left.\frac{\partial \theta_{1}}{\partial x}\right|_{x=0}=\left.\frac{\partial \theta_{2}}{\partial x}\right|_{x=0}=0 \quad \text { at the fin tip, } \\
& \theta_{0}(1)=1, \quad \theta_{1}(1)=0, \quad \theta_{2}(1)=0 \quad \text { at the base. }
\end{aligned}
$$

Solving these equations we firstly obtain $\theta_{0}(x)=1$ given that the boundary condition given in (16) needs to be satisfied. In Moitsheki and Harley [7], the solutions for small $\mathscr{M}$ flatten and the temperature at $x=0$ tends to 1 . As such the solution $\theta_{0}(x)=1$ is a reasonable approximation. We solve (13) and (14) via MATHEMATICA and obtain the following solution:

$$
\begin{aligned}
& \theta(x) \\
& =1+\left(\frac{x^{2}-1}{2(\beta+1)}\right) \mathscr{M}^{2} \\
& \quad+\left(\frac{\left(x^{2}-1\right)\left(\left(x^{2}-5\right)(1+n(1+\beta))-2 \beta\left(x^{2}-1\right)\right)}{24(1+\beta)^{3}}\right) \mathscr{M}^{4} .
\end{aligned}
$$

This solution verifies the notion that the length of the fin has a proportional relationship to the thermogeometric parameter. In assuming that $\mathscr{M} \ll \epsilon$, we found an asymptotic solution which when plotted has a high temperature at the fin tip-see Figure 2. Simplistically our results seem to indicate that when the thermo-geometric parameter has a small value, then the fin is short which only increases the temperature profile of the entire fin.

It needs to be noted that the solution does not allow for the case $\beta=-1$, even though the assumption that one has a decreasing gradient seems a plausible one. Were one to conduct the same calculations done to obtain the above solution (17) with an initial choice of $\beta=-1$, one would obtain a contradiction when separating according to powers of the thermo-geometric parameter such that for $\mathscr{M}^{2}:-1=0$.
As such, this asymptotic solution would not be valid for the instance when $\beta=-1$.

3.2. Boundary Layer Analysis: $\mathscr{M} \gg \epsilon$. In this section we will consider the case where $\mathscr{M}$ is large and as such define it as $\mathscr{M}=1 / \epsilon$ for $\epsilon$ small. Thus we can write (9) as the following:

$$
\epsilon^{2} \frac{d}{d x}\left(f(x)(1+\beta \theta) \frac{d \theta}{d x}\right)=\theta^{n+1} .
$$

If $\epsilon$ is small as assumed then the solution can be approximated as $\theta \approx 0$. Since this solution, in and of itself, is not useful in understanding our problem we transform variables from $x \in[0,1]$ into $X \in[0, \infty]$ through the transformation $X=(1-x) / \delta$. Here we define $\delta$ as the distance from the point where the temperature ceases to be constant, say $x_{\star}$, and starts to increase as we move along the length of the fin, to $x_{N}=1$. We assume the solution to be constant except for the range $\left[x_{\star}, x_{N}\right]$, where $\delta=x_{N}-x_{\star}$. This transformation of the independent variable gives

$$
\frac{\epsilon^{2}}{\delta^{2}} \frac{d}{d X}\left(f(1-\delta X)(1+\beta \Theta) \frac{d \Theta}{d X}\right)=\Theta^{n+1},
$$

where $\Theta=\Theta(X)$ and the boundary conditions become $\Theta(0)=1$ and $\operatorname{Lim}_{X \rightarrow \infty} \Theta(X)=0$. In order to obtain a nontrivial solution, we require that at least two leading-order terms in this equation have the same order of magnitude. Balancing the terms we find that

$$
\frac{\epsilon^{2}}{\delta^{2}}=1,
$$

which means that $\epsilon=\delta=1 / \mathscr{M}$. In this manner we have obtained a relationship between the thermo-geometric parameter and $\delta$.

3.3. Remark. The method of asymptotics is effectively used here to inspect the behaviour of the solution for small $\mathscr{M}$. In 


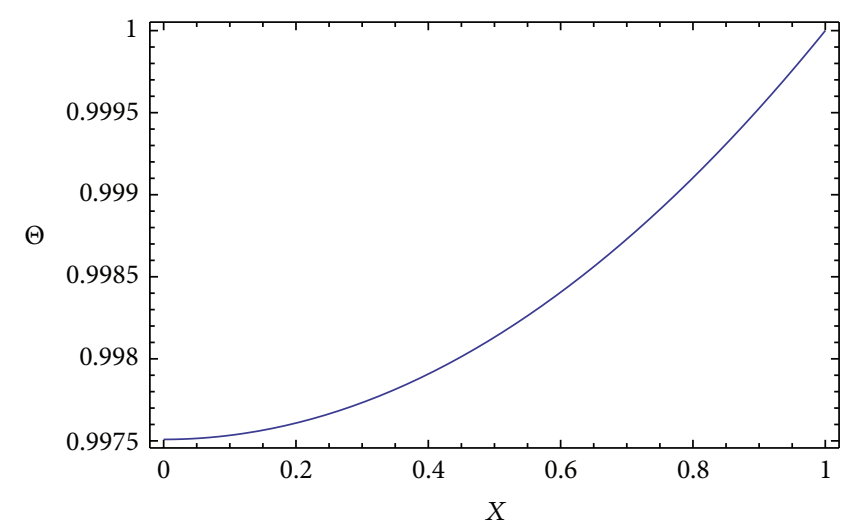

FIGURE 2: Plot of the asymptotic solution (17) with $\mathscr{M}=0.1$ and $\beta=n=1$.

previous work (see $[7,8]$ ) the thermo-geometric parameter was suspected to be proportional to the length of the fin, $L$. It was noticed that for small values of $\mathscr{M}$ there seemed to be unstable heat transfer in the fin-this was thought to be related to the fact that $\mathscr{M} \propto L$. Through our asymptotic analysis, we are now able to establish that for $\mathscr{M} \ll \epsilon$ the temperature at the fin tip (i.e., $x=0$ ) is quite high at 0.999975. This makes sense given that the value attached to $\theta_{0}(x)$ is one. This behaviour of the solution also supports the idea that $\mathscr{M} \propto L$ given that the overall temperature profile would increase if the fin were short.

For the case where $\mathscr{M} \gg \epsilon$ we found an inverse relationship between the thermo-geometric parameter and the distance $\delta$ defined above. This is again another indication of the proportional relationship between this parameter and the length of the fin, $L$, given that for large $\mathscr{M}, \delta$ is small which implies that $L$ must be large. The reason for this is twofold. Only when $L$ is large (1) would the temperature at the tip be $\ll \epsilon$ and (2) would the temperature gradient $\rightarrow 0$ for a long enough period of time to allow $\delta=x_{N}-x_{\star}$ to be small.

In order to investigate the dynamics of a system in which $\mathscr{M} \propto L$, we consider a dynamical system analysis. The purpose of this is to verify the results obtained thus far and also investigate the findings of $[7,8]$ with regards to possibly thermally unstable behaviour.

\section{Dynamical System Analysis}

As a means of further investigating the behaviour of the system we conduct a dynamical system analysis. We do this by rewriting (7) as a system of first-order ordinary differential equations through the transformations $\theta(x)=u(x)$ and $v(x)=d \theta / d x$ such that $d^{2} \theta / d x^{2}=v(d v / d \theta)$. This gives the following system:

$$
\begin{gathered}
\frac{d v}{d x}=\mathscr{M}^{2} u^{n+1}-\beta v^{2} \\
\frac{d u}{d x}=v(1+\beta u)
\end{gathered}
$$

with $u(1)=1$ and $v(0)=0$. We now use these equations and obtain the following equilibrium points:

$$
\begin{gathered}
z_{1}=(0,0), \quad z_{2}=\left(-\frac{1}{\beta},-\frac{\mathscr{M}(-1)^{(n+1) / 2}}{\beta^{(n+2) / 2}}\right), \\
z_{3}=\left(-\frac{1}{\beta}, \frac{\mathscr{M}(-1)^{(n+1) / 2}}{\beta^{(n+2) / 2}}\right),
\end{gathered}
$$

and nullclines which are curves drawn in the phase portrait along which one of the state variables does not change in time

$$
v=u^{3 / 2}, \quad v=-u^{3 / 2}
$$

The Jacobian matrix $J$ for the system (21) is given by

$$
J(u, v)=\left[\begin{array}{cc}
\beta v & 1+u \beta \\
\mathscr{M}^{2}(n+1) u^{n} & -2 \beta v
\end{array}\right] .
$$

To be able to do a phase plane analysis of the relevant equation we need to linearise the system. This is done via the calculation of the Jacobian (24), where the elements in $J$ need to be linear with respect to $v$. We now consider the Jacobian (24) for the equilibrium points given in (22). Firstly, we consider the point $z_{1}=(0,0)$ which produces the following Jacobian equation:

$$
J\left(u_{1}, v_{1}\right)=\left[\begin{array}{ll}
0 & 1 \\
0 & 0
\end{array}\right] .
$$

This in turn produces the eigenvalues

$$
\lambda_{1}^{(1)}=0, \quad \lambda_{2}^{(1)}=0
$$

The phase diagrams produced for this equilibrium point - to be seen in Figure 3-indicate that the point is a centre which is neutrally stable for $u<0$ joined to a saddle which is unstable for $u>0$. Furthermore, given that the Jacobian matrix has zero eigenvalues this critical point is a nonhyperbolic and degenerate singular point. More precisely, the linear part of the vector field can now be said to be doubly degenerate and the reduced system on the center manifold is 


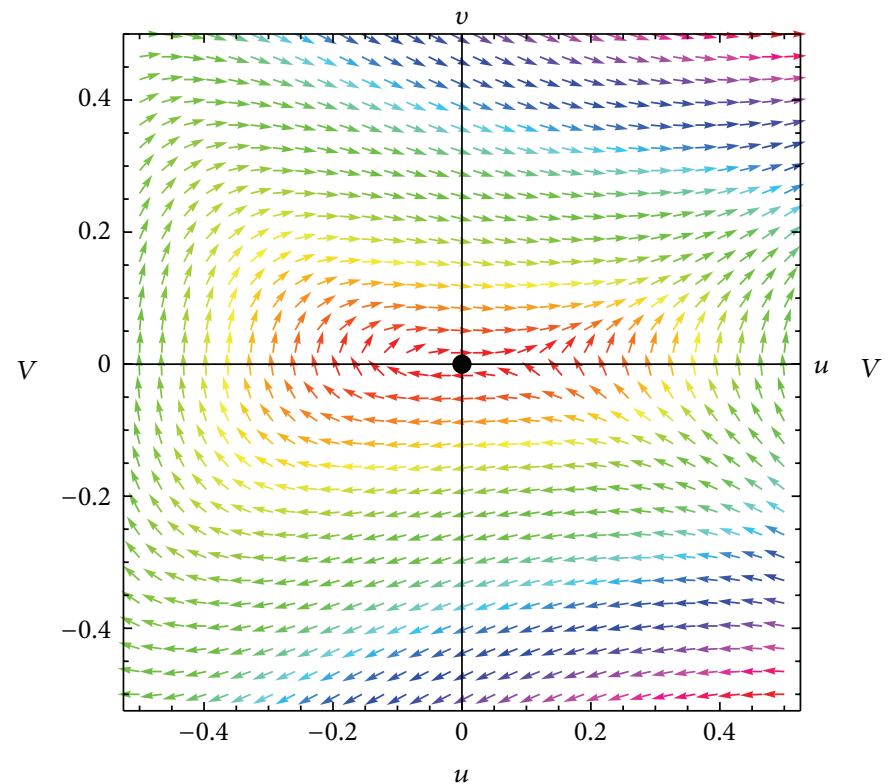

(a)

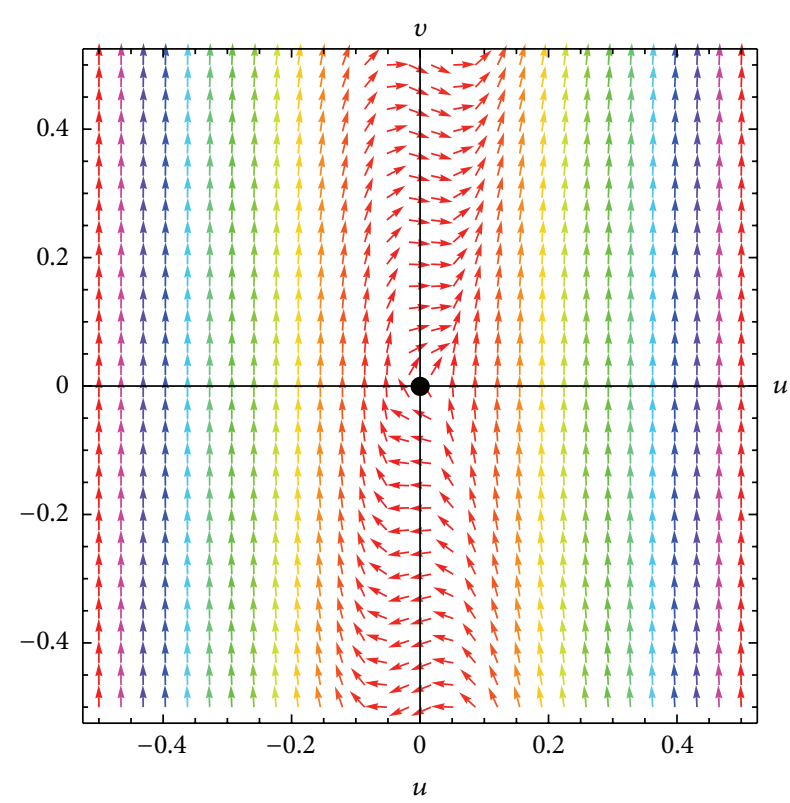

(b)

Figure 3: Plot of the phase trajectories for system (25) with $\mathscr{M}=1$ (a) and $\mathscr{M}=10$ (b) with $\beta=n=1$.

two-dimensional [12]. The classification and unfolding of this (nilpotent) type was done simultaneously and independently by Takens [13] and Bogdanov [14]. The normal form of the case (25) can be obtained as per Takens [13] and done in Guckenheimer and Holmes [12] and written as

$$
\begin{gathered}
\dot{u}=v+a u^{2}, \\
\dot{v}=b u^{2} .
\end{gathered}
$$

Takens [13] went on to show that (27) determines the local topological type of any vector field

$$
\begin{gathered}
\dot{u}=v+a u^{2}+\mathcal{O}\left(|x, y|^{3}\right), \\
\dot{v}=b u^{2}+\mathcal{O}\left(|x, y|^{3}\right)
\end{gathered}
$$

provided that $b \neq 0$. The predominant tool is a technique called blowing-up. Singular changes to the coordinates are introduced which expand the degenerate fixed points into circles containing a finite number of fixed points. If these are hyperbolic after the first blow-up, then the local flow near the circle, and, hence, near the original fixed point is stable with respect to higher-order terms. In our case three blow-ups are required for the transformed vector field to become stable. The first transformation is to polar coordinates given by $u=$ $r \cos \theta, v=r \sin \theta$, and still leaves us with a degenerate vector field. After two further blow-ups however- $(r, \theta) \rightarrow(\rho, \phi)$ defined by $\theta=\rho \cos \phi, r=\rho \sin \phi$, and $(\rho, \phi) \rightarrow(\eta, \psi)$ defined by $\phi=\eta \cos \psi, \rho=\eta \sin \psi$-the following vector field is obtained [12]:

$$
\begin{gathered}
\dot{\eta}=\eta^{2}\left(-b \cos ^{3} \psi+2 \cos ^{2} \psi \sin \psi\right. \\
\left.-\sin ^{3} \psi+b \cos \psi \sin ^{2} \psi \cdots\right), \\
\dot{\psi}=\eta\left(b \cos ^{3} \psi \sin \psi-3 \cos \psi \sin ^{2} \psi+b \cos ^{2} \psi \sin \psi \cdots\right) .
\end{gathered}
$$

Division of the vector field by $\eta$ will leave the phase portrait of (29) unaffected (except possibly at $\eta=0$ ) and since the common factor $\eta$ occurs in both components we may consider the "divided out" vector field given by the following:

$$
\begin{gathered}
\dot{\eta}=\eta\left(-b \cos ^{3} \psi+2 \cos ^{2} \psi \sin \psi\right. \\
\left.-\sin ^{3} \psi+b \cos \psi \sin ^{2} \psi \cdots\right), \\
\dot{\psi}=b \cos ^{3} \psi \sin \psi-3 \cos \psi \sin ^{2} \psi+b \cos ^{2} \psi \sin \psi \cdots,
\end{gathered}
$$

which has six hyperbolic fixed points at $\eta=0, \psi=0, \pi / 2, \pi$, $3 \pi / 2$, and $\psi=\arctan (2 b / 3)$. Hence the flow of (30) is stable to small (higher-order) perturbations and consequently the flow of (29) near $\eta=0$ is similarly stable. If we now "blowdown" three times $(\eta, \psi) \rightarrow(\rho, \phi) \rightarrow(r, \theta) \rightarrow(x, y)$, we are able to conclude that the flow of (27) near the degenerate fixed point $(x, y)=(0,0)$ is indeed stable with respect to the addition of (small) higher-order terms provided that $b \neq 0$. 


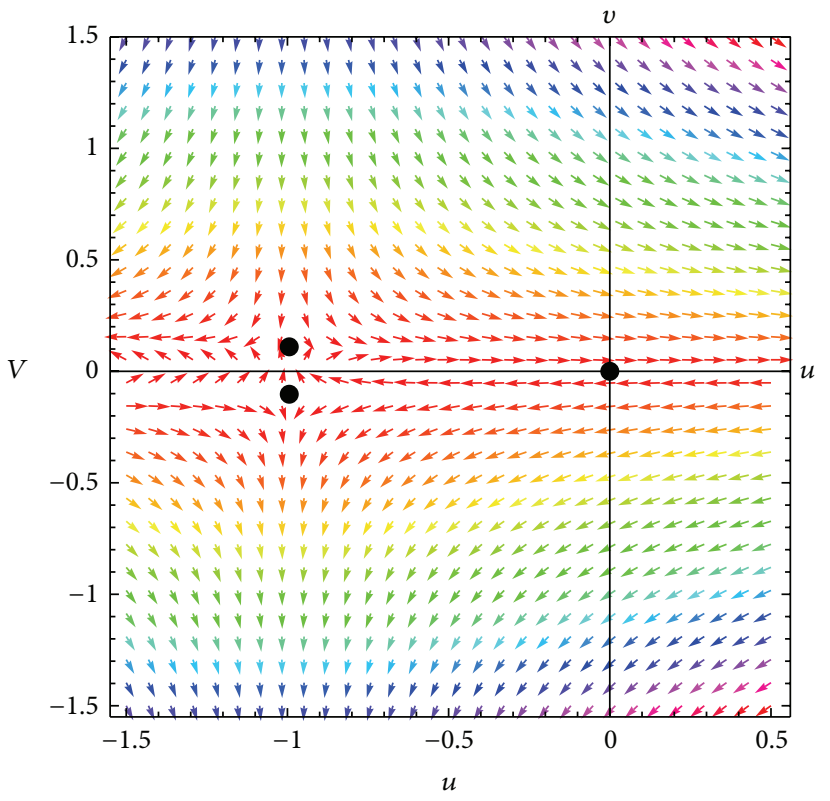

(a)

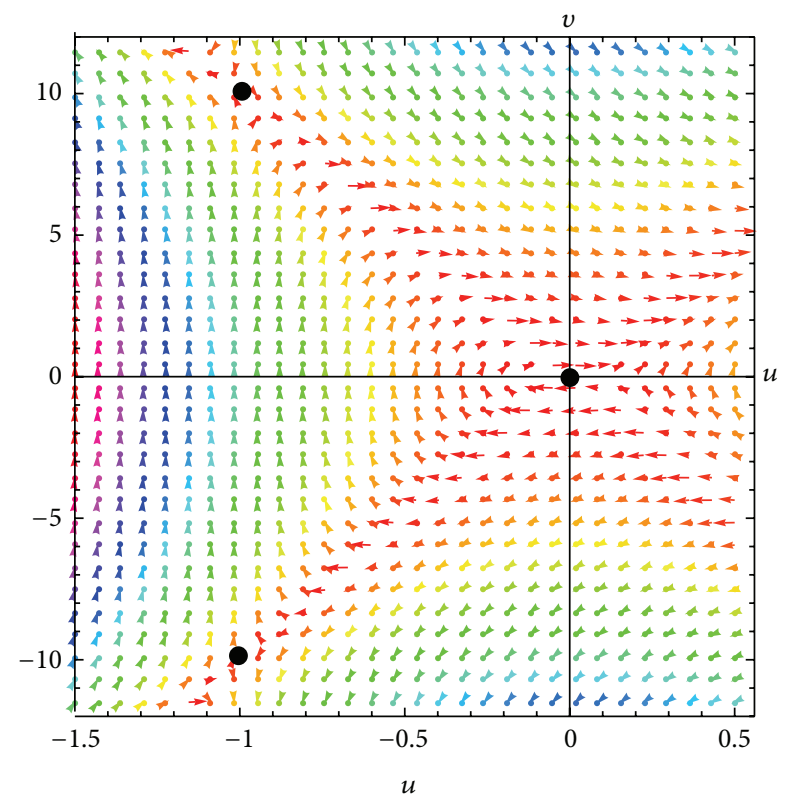

(b)

FIgURE 4: Plot of the phase trajectories for system (25) with $\mathscr{M}=0.1$ (a) and $\mathscr{M}=10$ (b) with $\beta=n=1$.

We now turn to our other two equilibrium points. Evaluating $J$ at $z_{2}$ produces the following:

$$
J\left(u_{2}, v_{2}\right)=\left[\begin{array}{cc}
-\mathscr{M}(-1)^{(n+1) / 2} \beta^{(-n / 2)} & 0 \\
\mathscr{M}^{2}(n+1)(-\beta)^{-n} & 2 \mathscr{M}(-1)^{(n+1) / 2} \beta^{(-n / 2)}
\end{array}\right],
$$

with eigenvalues

$$
\begin{aligned}
& \lambda_{1}^{(2)}=-M(-1)^{(n+1) / 2} \beta^{(-n / 2)}, \\
& \lambda_{2}^{(2)}=2 \mathscr{M}(-1)^{(n+1) / 2} \beta^{(-n / 2)} .
\end{aligned}
$$

When we consider $J$ at $z_{3}$ we find that

$$
\begin{aligned}
& J\left(u_{3}, v_{3}\right) \\
& \quad=\left[\begin{array}{cc}
\mathscr{M}(-1)^{(n+1) / 2} \beta^{(-n / 2)} & 0 \\
\mathscr{M}^{2}(n+1)(-\beta)^{-n} & -2 \mathscr{M}(-1)^{(n+1) / 2} \beta^{(-n / 2)}
\end{array}\right],
\end{aligned}
$$

where the eigenvalues are

$$
\begin{gathered}
\lambda_{1}^{(3)}=-2 M(-1)^{(n+1) / 2} \beta^{(-n / 2)}, \\
\lambda_{2}^{(3)}=\mathscr{M}(-1)^{(n+1) / 2} \beta^{(-n / 2)} .
\end{gathered}
$$

For these two equilibrium points we find that we have two cases to consider:

(a) eigenvalues with no zero or purely imaginary eigenvalues,

(b) eigenvalues which are purely imaginary.
In the first instance we turn to the Hartman-Grobman theorem which states that if $J=D f(\bar{x})$ has no zero or purely imaginary eigenvalues, then there is a homeomorphism $h$ defined on some neighbourhood $U$ of $\bar{x}$ in $\mathbb{R}^{n}$ locally taking orbits of the nonlinear flow (21) to those of the linear flow (31)-(33) $[12,15]$. The homeomorphism preserves the sense of orbits and can also be chosen to preserve parametrization by time.

For case (a) where $J$ has no eigenvalues with zero real part, $\bar{x}$ is called a hyperbolic or nondegenerate fixed point and the asymptotic behaviour of solutions near it (and hence its stability type) is determined by the linearisation. For this to be the case we must have that $(n+1) / 2$ is even. In Figure 4 we obtain eigenvalues which produce unstable saddle points; these results are similar to those obtained in Harley and Moitsheki [8] where the system was found to have two unstable saddle points for $n=1$. As $\mathscr{M}$ increases this behaviour becomes more visually apparent, however when the thermo-geometric parameter takes on a small value it becomes more difficult to analyse the stability of the system visually.

If however, as in the case of (b), any one of the eigenvalues has zero real part, then stability cannot be determined by linearisation. Thus, given that the linearised system does not describe the nonlinear system, we consider phase diagrams for specific values of our parameters as a means of understanding the dynamics of the system. If we choose $n=2$ we end up with the case in question and obtain the following equilibrium points: $z_{1}=(0,0), z_{2}=(-1, i)$ and $z_{3}=(-1,-i)$, where $i=\sqrt{-1}$. In Figure 5 we see the phase diagrams for the system (25) for $\mathscr{M}=0.1,1$, and 10 . The real parts of the points $z_{2}$ and $z_{3}$ lie on top of each other to the left of the origin. 


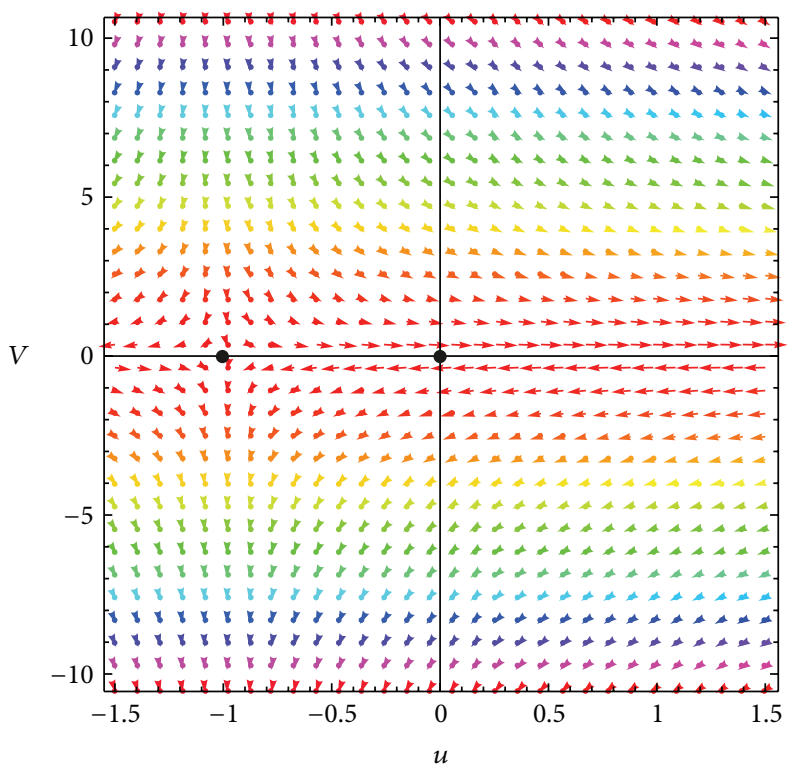

(a)

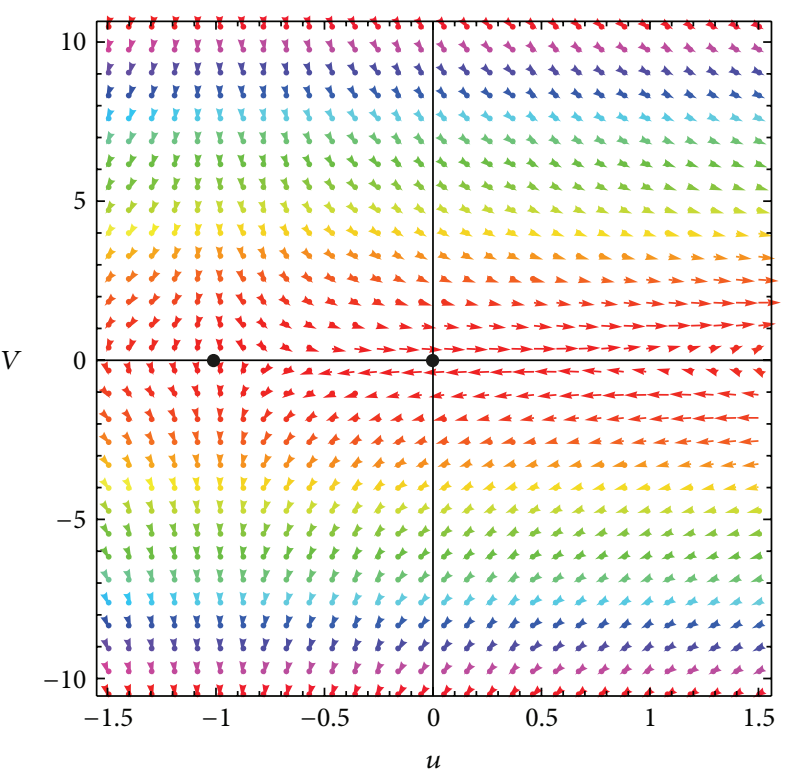

(b)

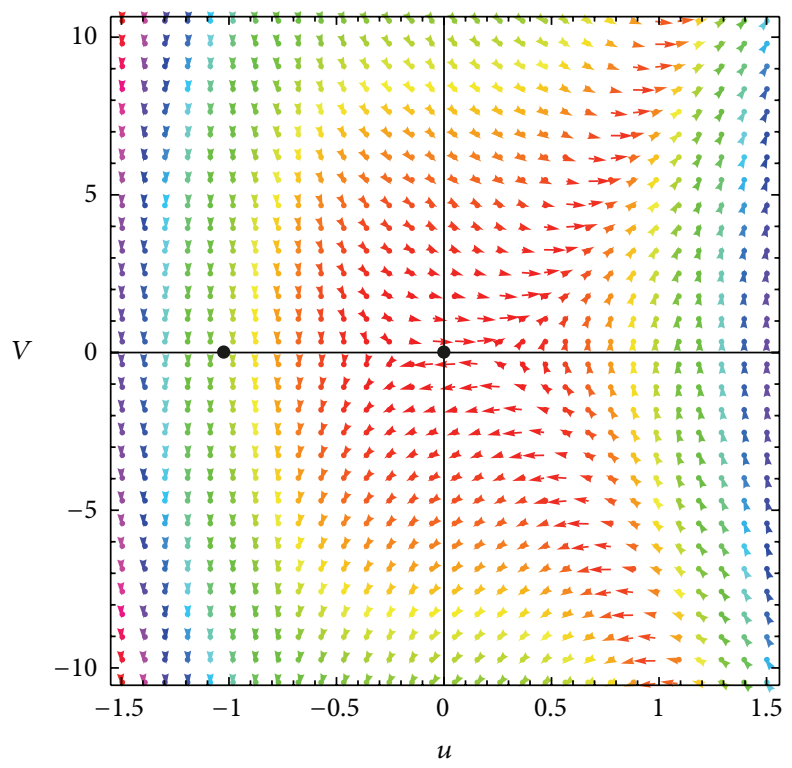

(c)

Figure 5: Plot of the phase trajectories for system (25) with $\mathscr{M}=0.1$ (a), $\mathscr{M}=1$ (b), and $\mathscr{M}=10$ (c) with $\beta=1$ and $n=2$.

For $\mathscr{M}=0.1$ the dynamics of $z_{2}$ and $z_{3}$ are akin to that of a saddle point. The representation of the equilibrium point $z_{1}=(0,0)$ seems to be influenced by the points $z_{2}$ and $z_{3}$ and behaves in a manner corresponding with what is seen when considering some linear systems [16]. In fact what we see visually corresponds to the case when $z_{1}$ only has one linearly independent eigenvector in which case the line determined by the eigenvector consists of fixed points, and all other solution curves are parallel to this line, moving in opposite directions on either side of the line [16]. This behaviour is local and is consistent with the analysis conducted above in which the degenerate point $z_{1}$ was found to be stable with respect to the addition of (small) higher-order terms.
When $\mathscr{M}=1$ we notice that $z_{2}$ and $z_{3}$ seem to create a centre (neither attracting nor repelling) for $u<-1$ attached to a saddle point for $u>-1$. However, when $\mathscr{M}$ increases to 10 the points $z_{2}$ and $z_{3}$ are now positioned on top of each other which causes the system to behave in a manner indicative of a plane of equilibria. This means that nothing moves and every solution curve is a point with the form $x=x_{0}$ and $\theta=\theta_{0}$.

As such, for these values of $\mathscr{M}$ the system does not behave in a stable manner around $z_{2}$ and $z_{3}$-at best we find neutrally stable behaviour. Even if a solution were to start sufficiently near these equilibrium points, it would not necessarily remain bounded. Furthermore, given that 
as $t \rightarrow \infty$ the solutions may not remain close or actually tend to the equilibrium points we cannot claim that the critical points are asymptotically stable either.

\section{Concluding Remarks}

In this paper, we were able to obtain an asymptotic solution which clarifies the behaviour of the system when $\mathscr{M} \ll \epsilon$. The solution curve also verified our suspicions that the thermogeometric parameter is proportional to the length of the fin. Furthermore, when we consider large $\mathscr{M}$ - that is, when $\mathscr{M} \gg$ $\epsilon$-we found that $\mathscr{M} \propto L$ via a boundary layer analysis. This justifies the work conducted in $[7,8]$ with regards to this relationship.

As a means of further investigating the effects of $\mathscr{M}$ on the dynamics of the system we conducted a dynamic system analysis. In doing so we found that when the temperature gradient is zero-this is true specifically at the tip of the fin where $\left.\theta^{\prime}\right|_{x=0}=0$-the system is in fact stable, with respect to the addition of (small) higher-order terms, even though the behaviour exhibited may indicate otherwise. Furthermore, the behaviour of the other equilibrium points is that of unstable saddle points when $n$ is odd, which according to the Hartman-Grobman theorem can be ascertained via a consideration of the linearised system in this instance. The system maintains unstable (or neutrally stable when $\mathscr{M}=$ 0.1 ) behaviour when $n$ is even - the cases of $\mathscr{M}=4$ and 6 were also considered - as can be witnessed when considering the phase diagrams. Lastly, we find that while it is quite a complex endeavour to investigate the stability at the tip of the fin-that is, when $\theta^{\prime}=0$-it is even more complicated to consider the dynamics of the system when $\mathscr{M}$ becomes small, that is, when the fin is short.

\section{Acknowledgments}

C. Harley, thanks H. Ockendon, C. Hall, and C. Please for useful discussion and acknowledges support from the National Research Foundation, South Africa, under Grant no. 79184. Furthermore, this paper was made possible (in part) by a grant from the Carnegie Corporation of New York. The statements made and views expressed are, however, solely the responsibility of the author.

\section{References}

[1] A. D. Kraus, A. Aziz, and J. Welty, Extended Surface Heat Transfer, John Wiley and Sons, New York, NY, USA, 2001.

[2] B. Sunden and P. J. Heggs, Eds., Recent Advances in Analysis of Heat Transfer for Fin Type Surfaces, WIT Press, Boston, Mass, USA, 2000.

[3] F. Khani, M. Ahmadzadeh Raji, and H. Hamedi Nejad, "Analytic solutions and efficiency of the nonlinear fin problem with temperature-dependent thermal conductivity and heat transfer coefficient," Communications in Nonlinear Science and Numerical Simulation, vol. 14, pp. 3327-3338, 2009.
[4] F. Khani, M. Ahmadzadeh Raji, and H. Hamedi-Nezhad, "A series solution of the fin problem with a temperaturedependent conductivity," Communications in Nonlinear Science and Numumerical Simulation, vol. 14, no. 7, pp. 3007-3017, 2009.

[5] A. A. Joneidi, D. D. Ganji, and M. Babaelahi, "Differential transformation method to determine fin efficiency of convective straight fins with temperature dependent thermal conductivity," International Communications in Heat and Mass Transfer, vol. 36, pp. 757-762, 2009.

[6] A. Aziz and T. Y. Na, "Periodic heat transfer in fins with variable thermal parameters," International Journal of Heat and Mass Transfer, vol. 24, no. 8, pp. 1397-1404, 1981.

[7] R. J. Moitsheki and C. Harley, "Transient heat transfer in longitudinal fin of various profiles with temperature-dependent thermal conductivity and heat transfer coefficient," Pramana: Journal of Physics, vol. 77, no. 3, pp. 519-532, 2011.

[8] C. Harley and R. J. Moitsheki, "Numerical investigation of the temperature profile in a rectangular longitudinal fin," Nonlinear Analysis: Real World Applications, vol. 13, no. 5, pp. 2343-2351, 2012.

[9] R. J. Moitsheki, T. Hayat, and M. Y. Malik, "Some exact solutions of the fin problem with a power law temperature-dependent thermal conductivity," Nonlinear Analysis: Real World Applications, vol. 11, no. 5, pp. 3287-3294, 2010.

[10] R. J. Moitsheki, "Steady one-dimensional heat flow in a longitudinal triangular and parabolic fin," Communications in Nonlinear Science and Numerical Simulation, vol. 16, no. 10, pp. 3971-3980, 2011.

[11] H. C. Ünal, "The effect of the boundary at a fin tip on the performance of the fin with and without internal heat generation," International Journal of Heat and Mass Transfer, vol. 31, no. 7, pp. 1483-1496, 1998.

[12] J. Guckenheimer and P. Holmes, Nonlinear Oscillations, Dynamical Systems, and Bifurcations of Vector Fields, vol. 42 of Applied Mathematical Sciences, Springer, New York, NY, USA, 1983.

[13] F. Takens, "Singularities of vector fields," Publications Mathématiques de l'IHÉS, no. 43, pp. 47-100, 1974.

[14] V. I. Bogdanov, "Lectures on bifurcations and versal families," Russian Mathematical Surveys, vol. 27, no. 5, pp. 54-123, 1972.

[15] P. Hartman, Ordinary Differential Equations, John Wiley \& Sons, New York, NY, USA, 1964.

[16] B. Hasselblatt, "Phase portraits of linear systems," http://citeseerx.ist.psu.edu/viewdoc/summary?doi=10.1.1.184.8666. 


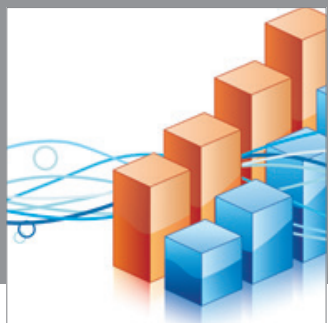

Advances in

Operations Research

mansans

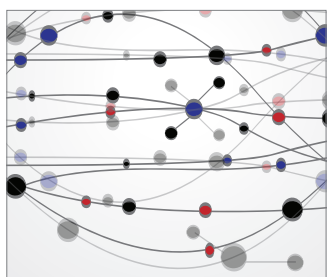

The Scientific World Journal
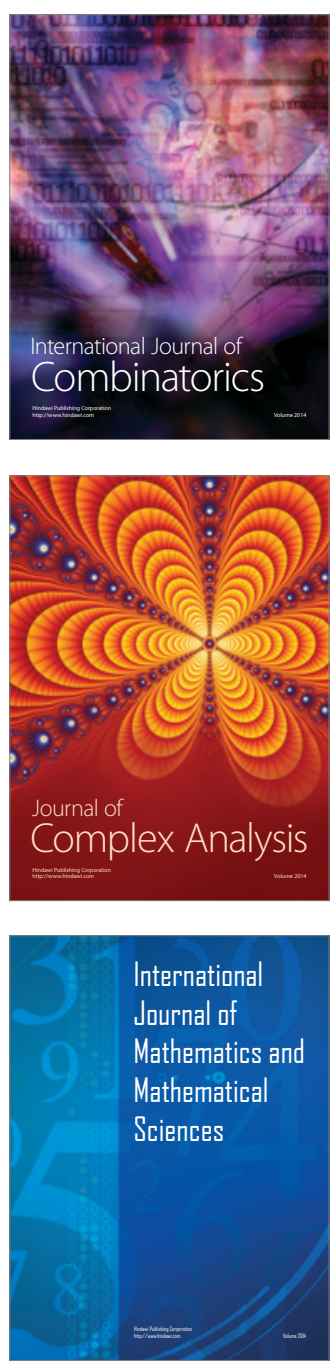
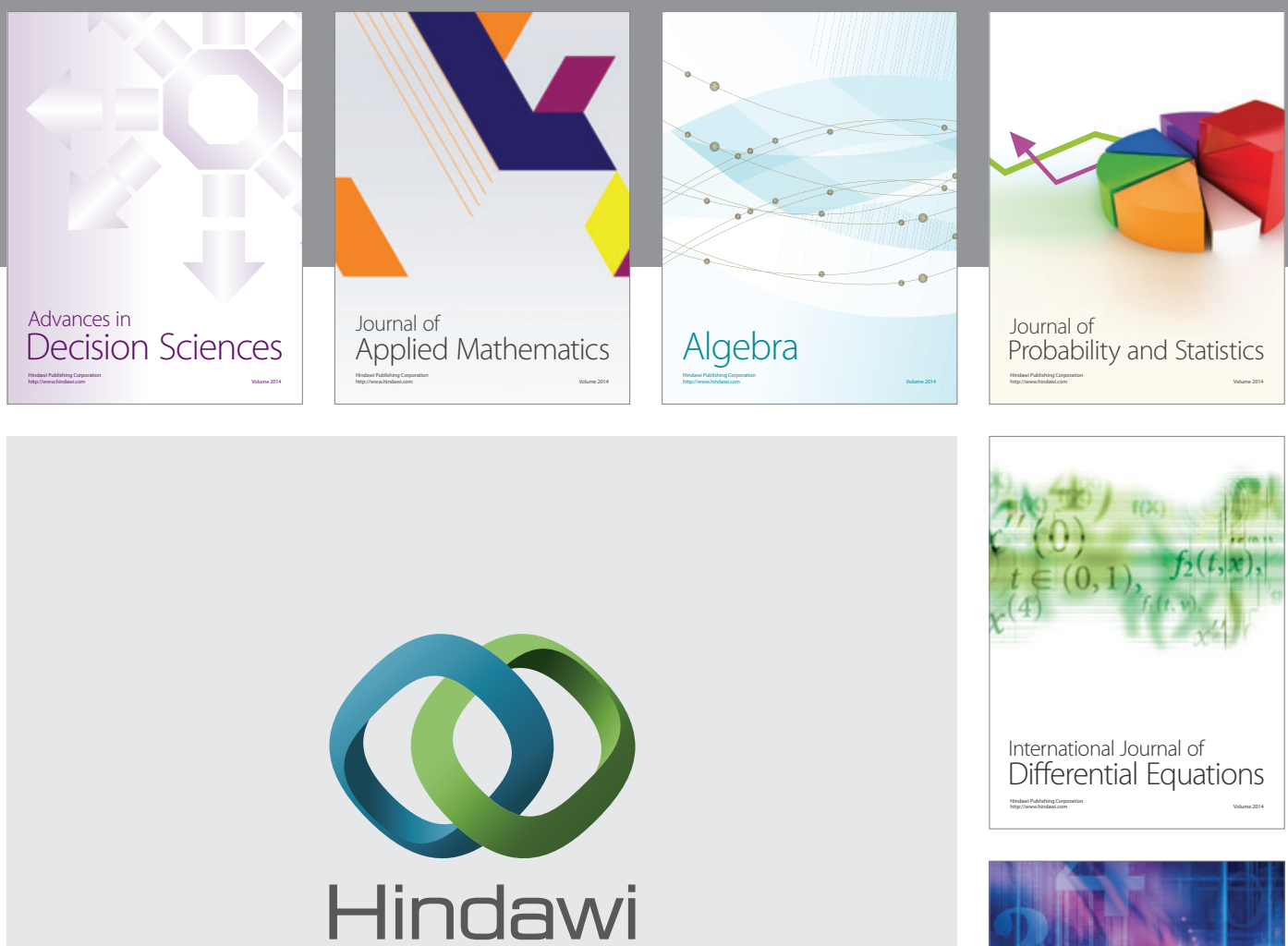

Submit your manuscripts at http://www.hindawi.com
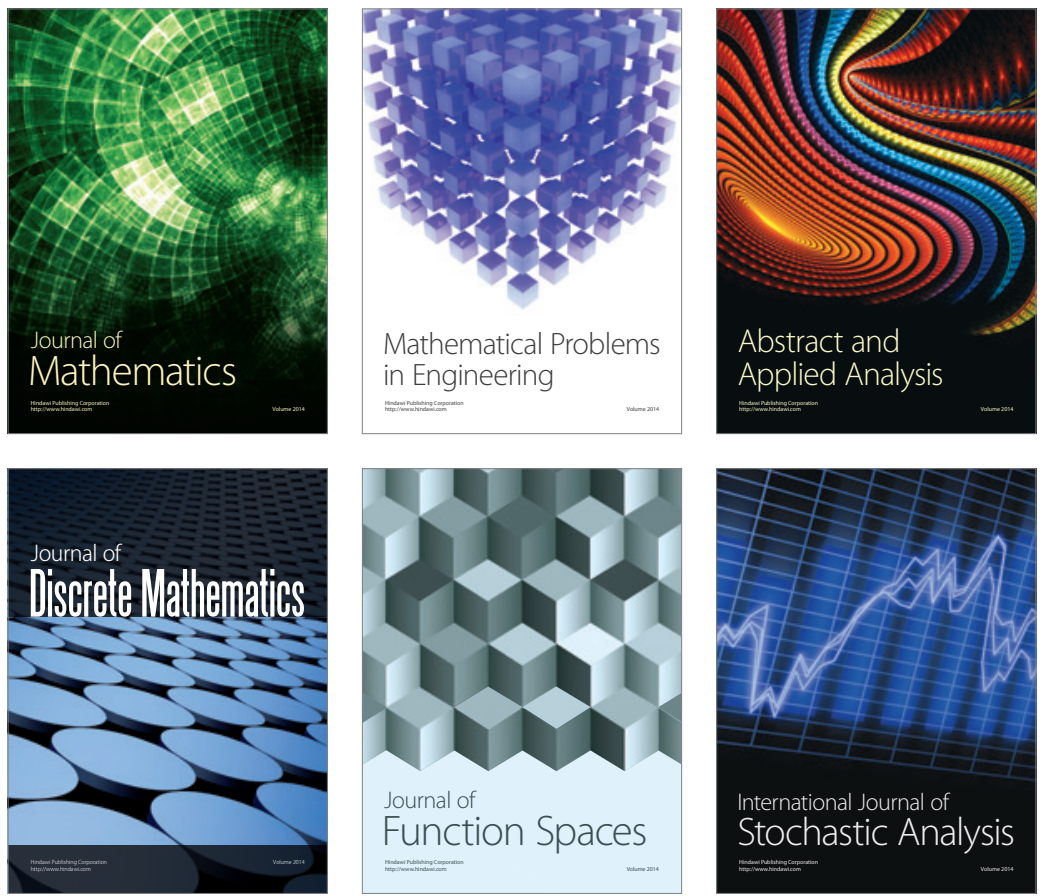

Journal of

Function Spaces

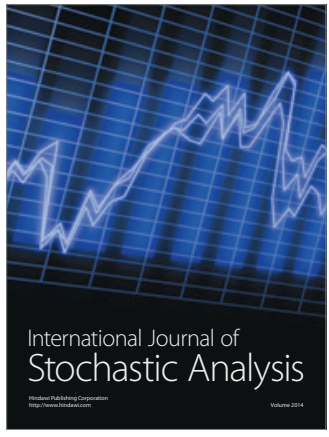

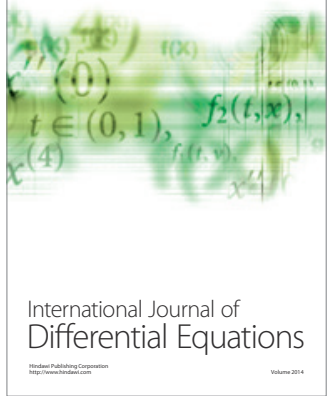
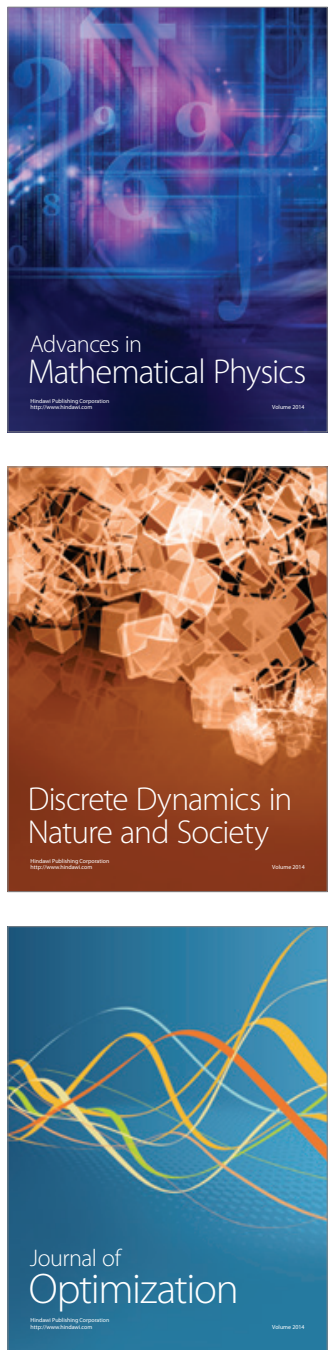